\title{
Il valore culturale del patrimonio naturale nella promozione dello sviluppo sostenibile
}

\author{
Marialuisa Saviano
}

\begin{abstract}
Obiettivo del paper: Il presente lavoro intende fornire un contributo alla definizione di possibili modelli di valorizzazione del patrimonio culturale in unottica di sostenibilità economica, sociale e ambientale.

Metodologia: Muovendo da una visione dellambiente quale componente naturalistica del patrimonio culturale, il lavoro è sviluppato avvalendosi del contributo interpretativo degli schemi generali dell'Approccio Sistemico Vitale e avendo come riferimento il caso delle Riserve della Biosfera del Programma "Man and the Biosphere" (MaB) dell'UNESCO.

Risultati: Il contributo delinea $i$ tratti di un possibile modello di valorizzazione culturale del patrimonio naturale in unottica di sostenibilità, derivandoli dallanalisi del modello 'MaB' che mette in luce il valore culturale di un sito, quindi di un territorio, quale risultante del dinamico evolvere del rapporto tra uomo e natura, superando la tradizionale visione oggettiva del valore. In tale prospettiva, la valorizzazione si fonda sulla capacità di realizzare nel territorio modelli di sviluppo sostenibile, che concilino esigenze economiche, sociali e ambientali, in un'unitaria ottica di sostenibilità.

Limiti della ricerca: Il lavoro propone l'elaborazione concettuale di un caso di studio per trarne stimoli interpretativi le cui potenzialità possono essere ulteriormente esplorate attraverso studi empirici.

Implicazioni pratiche: Il contributo offre una chiave di lettura del tema "Heritage, management e impresa" che, evidenziando la centralità dell'impresa e il contributo del management, appare foriera di interessanti sviluppi.

Originalità del paper: L'approccio interpretativo sviluppato integra, in unottica unitaria di sostenibilità, prospettive tradizionalmente disallineate nel guardare al patrimonio, alla valorizzazione e alle sfide della sostenibilità.
\end{abstract}

Parole chiave: patrimonio naturale; valore culturale; approccio sistemico vitale; valorizzazione; sostenibilità; sviluppo sostenibile

Purpose of the paper: This paper aims to contribute to the definition of possible models for the enhancement of cultural heritage from the perspective of economic, social and environmental sustainability.

Methodology: Viewing the environment as a naturalistic component of cultural heritage, this work is developed by benefitting from the interpretative tools of the general schemes of the Viable System Approach and makes reference to the case of the Biosphere Reserves of the UNESCO 'Man and the Biosphere' (MaB) Program.

Findings: Our contribution puts forward a model for enhancing cultural heritage from the perspective of sustainability. The analysis of the 'MaB' model brings to light 
sinergie Vol. 34, N. 99, 2016

the value of a site as a result of the dynamic evolution of the relationship between man and nature, thus aiming to overcome a traditional objective view of value. In our research, value is understood in terms of the ability to implement models of sustainable development which integrate economic, social and environmental perspectives in a single sustainability framework.

Research limits: The paper proposes the conceptual elaboration of a case study whose potential can be explored through further empirical research.

Practical implications: The proposed interpretation of the theme 'Heritage, management and business' suggests interesting developments by highlighting the key role of the firm and the contribution of management.

Originality of the paper: The interpretive approach developed in the paper brings traditionally misaligned economic, social and environmental perspectives under a unified framework focused on sustainability.

Key words: natural heritage; cultural value; viable systems approach; enhancement; sustainability; sustainable development

\section{Introduzione}

Il presente contributo muove da una visione dell'ambiente quale componente naturalistica del patrimonio culturale, sviluppata nellambito del filone di studi sull'approccio sistemico in economia d'impresa (Golinelli, 2000, 2011, 2012, 2014, 2015; Barile, 2000, 2008, 2009, 2012, 2014; Montella, 2012, 2014, 2015), ed elabora una proposta interpretativa utile alla definizione di possibili modelli di valorizzazione del patrimonio culturale in unottica di sostenibilità economica, sociale e ambientale.

La prospettiva adottata (Montella, 2009; Tamma, 2010; Golinelli, 2015) mette in luce il valore culturale del patrimonio naturale come 'testimonianza di civiltà in quanto risultante del dinamico evolvere del rapporto tra uomo e natura. In tale prospettiva, emergono elementi di riflessione utili ai fini allo sviluppo di un possibile modello di valorizzazione del patrimonio in ottica di sostenibilità. Emergono, inoltre, spunti interessanti per una riflessione di più ampia portata sulle condizioni di un armonico rapporto tra economia, ambiente e società, che vedono riaffermati la centralità dell'impresa e il contributo del management. Si perviene, così, a una possibile chiave di lettura del tema "Heritage, management e impresa" che appare foriera di interessanti sviluppi.

La riflessione è condotta avendo come riferimento emblematico il caso delle aree protette - rilevante componente del patrimonio naturale - e individuando nel modello delle Riserve della Biosfera del programma $\mathrm{MaB}$ (Man and the Biosphere) dell'UNESCO - organizzazione fortemente impegnata nella tutela e valorizzazione del patrimonio in ottica di sostenibilità -, un possibile riferimento per la promozione dello sviluppo sostenibile nel e del territorio, fondato sull'integrazione degli obiettivi di conservazione e tutela in un'ampia strategia di valorizzazione.

L'UNESCO svolge un ruolo di notevole importanza nell'indirizzare verso il riconoscimento del valore culturale dei siti in ottica di sostenibilità, sollecitando un cambiamento di prospettiva nella valorizzazione del 
patrimonio che lo studioso di management ha teorizzato e formalizzato. La sollecitazione dell'UNESCO è di ampia portata: nel qualificarsi come United Nations Educational, Scientific and Cultural Organization, l'UNESCO
si occupa "di incoraggiare l'identificazione, la protezione e la salvaguardia nella promozione dello del patrimonio culturale e naturale: esso rappresenta la storia e l'identità di ciascun popolo e costituisce l'eredità del passato da trasmettere alle generazioni future" (UNESCO, 1970, p. 1). In un'unitaria prospettiva educativa, scientifica e culturale, dunque, l'UNESCO valorizza il patrimonio materiale, immateriale e naturale (UNESCO, 2001, art. 1) dell'umanità - sì noti - non dell'uomo, a sollecitare una visione che travalichi i confini nello spazio e nel tempo.

La prospettiva adottata, il modello analizzato e l'interpretazione proposta evidenziano come l'approccio imprenditoriale e manageriale possa indirizzare efficacemente verso percorsi di valorizzazione del patrimonio culturale, ampiamente inteso, conciliando esigenze economiche, sociali e ambientali in un'unitaria ottica di sostenibilità.

\section{L'ambiente come risultante del dinamico evolvere del rapporto tra uomo e natura}

Nell'accezione comune del termine, al concetto di 'ambiente' si è soliti associare l'aggettivo "naturale": l'ambiente naturale è oggetto di interesse diffuso, ma soprattutto di crescenti preoccupazioni, in particolare per quanto attiene alla conservazione della sua biodiversità in unottica di sostenibilità (Teofili e Clarino, 2008). Nel guardare all'ambiente in una prospettiva economica prevale l'accezione di bene ambientale o patrimonio naturale e il focus si sposta dalle problematiche della conservazione e della tutela a quelle della valorizzazione, chiamando in causa l'economista d'impresa quale studioso di modelli e tecniche di management che dotano il decisore delle capacità e competenze necessarie ai fini della valorizzazione. La prospettiva dell'economista d'impresa esprime, nella più ampia ottica delle scienze sociali, il punto di vista di individui e organizzazioni che guardano all'ambiente come insieme di risorse da 'usare' o 'gestire' o, più correttamente, 'valorizzare' nei processi di produzione e consumo che si realizzano nei molteplici contesti dell'economia e della società (Massaroni e Rossi, 2007; Franch, 2010; Re, 2010; Golinelli, 2012, 2014; IUCN, 2013; Golinelli, 2015).

Ambiente, società ed economia sono, quindi, dimensioni (o prospettive) di una comune e unitaria fenomenica del reale, che è 'naturalmente' trasversale ai diversi ambiti (Elkington, 1998; Barile et al., 2013). Ciononostante, lo sviluppo specialistico delle scienze - ambientali, sociali ed economiche -, ha generato una 'innaturale' separazione che pone oggi gli studiosi, così come i decisori istituzionali e manageriali, di fronte al problema del recupero di una visione unitaria che, superando i confini disciplinari, crei un corpus di conoscenze interdisciplinari necessarie alla comprensione del rapporto tra impresa e ambiente (Cafferata, 1987; Ferrara, 1994) e, più in generale, tra uomo e natura in ottica di sostenibilità (Saviano, 2015).

Il crescente prevalere degli interessi economici ha condotto nel tempo a una sbilanciata configurazione del rapporto tra gli ambiti dell'economia, 
sinergie Vol. 34, N. 99, 2016

dell'ambiente e della società della nota rappresentazione della sostenibilità (Elkington, 1994), con un'alterazione di funzioni e ruoli delle diverse componenti dei sistemi socio-economici, cui è conseguita l'attuale condizione di squilibrio (Fig. 1). Prolungato nel tempo, tale squilibrato rapporto ha condotto alle contraddizioni, note a tutti, di un pianeta dove convivono ricchezza e povertà estrema, benessere e sofferenza, spreco e fame, uguaglianza e iniquità, accelerato progresso e profonda arretratezza (Meadows et al., 1972); il tutto nell'indifferenza dei più.

Fig. 1: Le tre dimensioni della sostenibilità tra teoria, realtà e cambiamento necessario
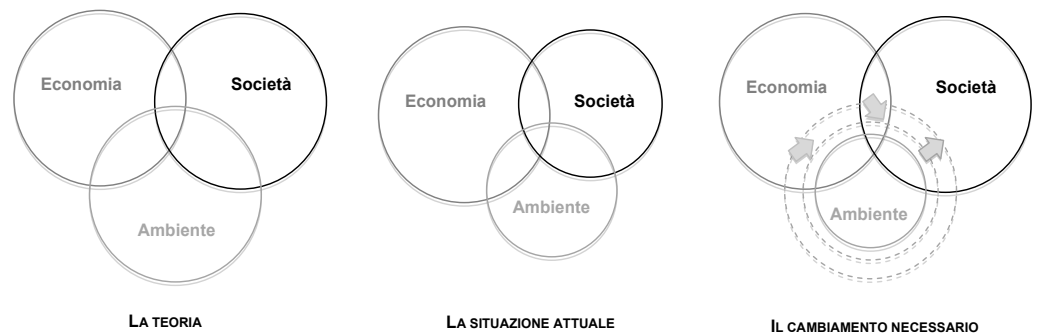

Fonte: Adattamento da IUCN, 2006, p. 2

In questo scenario, qual è il ruolo dell'impresa? E che cosa l'economista d'impresa, come studioso di management, è chiamato a fare?

Lo studioso di management, parallelamente al decisore d'impresa, vive i dilemmi di una transizione che fatica a compiersi, di aspirazioni declinate, spesso superficialmente, sul piano filosofico ma molto ardue a tradursi in operatività (Golinelli e Volpe, 2012).

Nella miopia di uno sguardo rivolto al qui e ora, agli oggetti, alle cose, ai confini, si è consumato un irresponsabile uso delle risorse ambientali ispirato da un'ottica di competitività in cui il gioco è inesorabilmente a somma zero, dove a fronte di chi vince cè sempre qualcuno che perde (Conner e Prahalad, 1996; De Toni e Comello, 2005). Un paradigma che ha indirizzato a lungo, e tuttora indirizza, le scelte dell'impresa, ma che già da tempo ha cominciato a vacillare dimostrando la fallacia di vantaggi individuali e di breve termine, quando conseguiti a danno di altri sistemi e, in particolare, dell'ambiente quale ecosistema.

Lorizzonte spazio-temporale dell'azione dell'impresa si è dovuto così ampliare progressivamente riconoscendo il fondamento di una responsabilità 'sociale', che deve conciliarsi con quella 'economica', ed estendendosi poi fino a quella 'ambientale' (Sciarelli, 2003, 2005), tanto nello spazio quanto nel tempo. In tale progressivo ampliamento di prospettiva, l'impresa familiarizza con la necessità di ascoltare le istanze di una più ampia varietà di stakeholder, attuali e potenziali, presenti e futuri, verso i quali assume precise responsabilità (Arrigo, 2008; Ciasullo e Trosi, 2013). Così, efficacia, efficienza, economicità, redditività, ecc. mostrano di non esaurire la gamma dei criteri guida nella gestione delle organizzazioni sociali e imprenditoriali, e un ulteriore criterio entra nel linguaggio del 
management modificandone profondamente l'ottica: la sostenibilità (Sen, 1986; Frey e Iraldo, 2009; Carroll and Buchholtz, 2014).

E in tale contesto che si ripropone, con la sempre rinnovata attualità di un problema mai risolto, la questione del rapporto dell'uomo (e dell'impresa) con l'ambiente naturale e dell'utilizzo dei beni comuni - la nota "tragedia dei beni comuni" (Hardin, 1968; Ostrom, 1999). Un ambiente che 'urla' la silenziosa sofferenza del degrado, della depauperazione, dell'inquinamento, dell'abuso, richiamando l'attenzione di policy maker, decisori d'impresa e comuni cittadini. In tale contesto, dunque, si palesa l'inadeguatezza delle misure finora adottate, che, pur mostrando una certa efficacia nel breve, spesso allontanano da ben più rilevanti traguardi di lungo termine, ad evidenza di una dinamica evolutiva avviata ma non compiuta che annuncia di dover andare ben oltre l'ottica della responsabilità e dell'accountability, riconoscendo la necessità di un cambiamento paradigmatico per un concreto avanzamento nella transizione verso la sostenibilità (Bauer, 1966; Maizza, 2013; Bebbington et al., 2014).

Sulla base di tale premessa, in quanto segue, avendo come riferimento il caso delle Riserve della Biosfera del Programma MaB dell'UNESCO, si rifletterà sul ruolo dell'impresa nel promuovere il recupero di un rapporto armonico tra uomo e natura, quindi tra società e ambiente, inteso come patrimonio da tutelare attraverso la valorizzazione (Barile e Saviano, 2012a, 2015). Si perverrà, così, ad una più ampia riflessione sul cammino verso la sostenibilità, in linea con diversi avanzamenti degli studi di management sia in ambito internazionale (Adebowale, 2002; Reynoso, 2009; Porter e Kramer, 2011; Pels et al., 2014; Crane et al., 2014; Reynoso et al., 2015) sia in ambito nazionale (Bonfanti, 2009; Frey e Iraldo, 2009; Rullani, 2010; 2012; Sarra e Mazzocchitti, 2014).

\section{Il sistema delle aree protette in Italia e le classificazioni UNESCO}

L'Italia è unica al mondo per varietà e densità di patrimonio materiale, immateriale e naturale ${ }^{1}$. Questa ricca dotazione ha posto seri problemi di conservazione dando impulso allo sviluppo di soluzioni normative, tecniche e tecnologiche per la 'tutela' di beni culturali e di sempre più ampie porzioni di territorio alle quali è riconosciuto un elevato valore naturalisticoambientale, quindi, nella nostra prospettiva, anche storico-culturale. La rilevanza di tali processi e la molteplicità di istituzioni governative (e non) coinvolte in iniziative altrettanto numerose e varie, unite a una non piena organicità dell'azione di governo, hanno generato una variegata gamma di definizioni, classificazioni, normative e programmi, che disegnano un quadro di riferimento a livello nazionale e internazionale spesso frammentato e talvolta incoerente (AA.VV., 2003).

A livello internazionale, un'area protetta è definita come "a clearly defined

1 Sul sistema delle aree protette in Italia, si rinvia al sito del Ministero dell'Ambiente e della Tutela del Territorio e del Mare, http://www.minambiente.it/pagina/areenaturali-protette.

Si veda, in particolare, il documento del 2013, Parchi Nazionali: dal capitale naturale alla contabilità ambientale, http://www.minambiente.it/sites/default/ files/archivio/comunicati/Attuazione_Interno_VI_bozza_okx7x_Layout_1.pdf.
Marialuisa Saviano

Il valore culturale

del patrimonio naturale

nella promozione dello

sviluppo sostenibile 
sinergie Vol. 34, N. 99, 2016

geographical space, recognised, dedicated and managed, through legal or other effective means, to achieve the long-term conservation of nature with associated ecosystem services and cultural values". (IUCN, 2008, p. 8). Il principale riferimento normativo italiano è la legge quadro 394 del 1991 che individua otto tipologie di aree naturali (Tab. 1) che compongono o, meglio, dovrebbero comporre, un 'sistema' di aree protette. In tale sistema, i Parchi nazionali, regionali e interregionali e le riserve naturali statali e regionali rappresentano la componente più rilevante sia da un punto di vista dimensionale, sia da un punto di vista gestionale, in quanto dotate di proprie strutture di governo.

Tab. 1: Tipologie di siti del Sistema Nazionale delle Aree Protette

\begin{tabular}{|c|c|}
\hline \multicolumn{2}{|r|}{ Il SISTEMA NAZIONALE DELLE AREE PROTETTE } \\
\hline Parchi Nazionali & $\begin{array}{l}\text { Sono costituiti da aree terrestri, fluviali, lacuali o marine che contengono uno } \\
\text { o più ecosistemi intatti o anche parzialmente alterati da interventi antropici, } \\
\text { una o più formazioni fisiche, geologiche, geomorfologiche, biologiche, di rilievo } \\
\text { internazionale o nazionale per valori naturalistici, scientifici, estetici, culturali, } \\
\text { educativi e ricreativi tali da richiedere l'intervento dello Stato ai fini della loro } \\
\text { conservazione per le generazioni presenti e future. }\end{array}$ \\
\hline $\begin{array}{l}\text { Parchi naturali } \\
\text { regionali } \\
\text { e interregionali }\end{array}$ & $\begin{array}{l}\text { Sono costituiti da aree terrestri, fluviali, lacuali ed eventualmente da tratti di } \\
\text { mare prospicienti la costa, di valore naturalistico e ambientale, che costituiscono, } \\
\text { nell'ambito di una o più regioni limitrofe, un sistema omogeneo, individuato } \\
\text { dagli assetti naturalistici dei luoghi, dai valori paesaggistici e artistici e dalle } \\
\text { tradizioni culturali delle popolazioni locali. }\end{array}$ \\
\hline Riserve naturali & $\begin{array}{l}\text { Sono costituite da aree terrestri, fluviali, lacuali o marine che contengono } \\
\text { una o più specie naturalisticamente rilevanti della flora e della fauna, ovvero } \\
\text { presentino uno o più ecosistemi importanti per la diversità biologica o per la } \\
\text { conservazione delle risorse genetiche. Le riserve naturali possono essere statali o } \\
\text { regionali in base alla rilevanza degli elementi naturalistici in esse rappresentati. }\end{array}$ \\
\hline $\begin{array}{l}\text { Zone umide di } \\
\text { interesse } \\
\text { internazionale }\end{array}$ & $\begin{array}{l}\text { Sono costituite da aree acquitrinose, paludi, torbiere oppure zone naturali o } \\
\text { artificiali d'acqua, permanenti o transitorie comprese zone di acqua marina la } \\
\text { cui profondità, quando c’è bassa marea, non superi i sei metri che, per le loro } \\
\text { caratteristiche, possono essere considerate di importanza internazionale ai sensi } \\
\text { della convenzione di Ramsar. }\end{array}$ \\
\hline $\begin{array}{l}\text { Altre aree naturali } \\
\text { protette }\end{array}$ & $\begin{array}{l}\text { Sono aree (oasi delle associazioni ambientaliste, parchi suburbani, ecc.) che } \\
\text { non rientrano nelle precedenti classi. Si dividono in aree di gestione pubblica, } \\
\text { istituite cioè con leggi regionali o provvedimenti equivalenti, e aree a gestione } \\
\text { privata, istituite con provvedimenti formali pubblici o con atti contrattuali quali } \\
\text { concessioni o forme equivalenti. }\end{array}$ \\
\hline $\begin{array}{l}\text { Zone di protezione } \\
\text { speciale (Zps) }\end{array}$ & $\begin{array}{l}\text { Designate ai sensi della direttiva 79/409/Cee, sono costituite da territori idonei } \\
\text { per estensione e/o localizzazione geografica alla conservazione delle specie di } \\
\text { uccelli di cui all'allegato I della direttiva citata, concernente la conservazione } \\
\text { degli uccelli selvatici. }\end{array}$ \\
\hline $\begin{array}{l}\text { Zone speciali di } \\
\text { conservazione }(\mathrm{Zsc})\end{array}$ & $\begin{array}{l}\text { Designate ai sensi della direttiva } 92 / 43 / \text { Cee, sono costituite da aree naturali, } \\
\text { geograficamente definite e con superficie delimitata, che: } \\
\text { a. contengono zone terrestri o acquatiche che si distinguono grazie alle loro } \\
\text { caratteristiche geografiche, abiotiche e biotiche, naturali o seminaturali } \\
\text { (habitat naturali) e che contribuiscono in modo significativo a conservare, } \\
\text { o ripristinare, un tipo di habitat naturale o una speciedella flora e della } \\
\text { fauna selvatiche di cui all'allegato I e II della direttiva } 92 / 43 / \mathrm{Cee} \text {, relativa } \\
\text { alla conservazione degli habitat naturali e seminaturali e della flora e della } \\
\text { fauna selvatiche in uno stato soddisfacente a tutelare la diversità biologica } \\
\text { nella regione paleartica mediante la protezione degli ambienti alpino, } \\
\text { appenninico e mediterraneo; } \\
\text { sono designate dallo Stato mediante un atto regolamentare, amministrativo } \\
\text { e/o contrattuale e nelle quali siano applicate le misure di conservazione } \\
\text { necessarie al mantenimento o al ripristino, in uno stato di conservazione } \\
\text { soddisfacente, degli habitat naturali e/o delle popolazioni delle specie } \\
\text { per cui l'area naturale è designata. Tali aree sono indicate come Siti di } \\
\text { importanza comunitaria (Sic). }\end{array}$ \\
\hline $\begin{array}{l}\text { Aree di reperimento } \\
\text { terrestri e marine }\end{array}$ & $\begin{array}{l}\text { Indicate dalle leggi 394/91 e 979/82, costituiscono aree la cui conservazione } \\
\text { attraverso l'istituzione di aree protette è considerata prioritaria. }\end{array}$ \\
\hline
\end{tabular}

Fonte: http://www.parks.it/ministero.ambiente/ 
Aree protette e relative superfici sono in crescita (Federparchi, 2009). A tale positivo risultato contribuisce generalmente una maggiore attenzione, soprattutto a livello internazionale, verso i temi dell'ambiente e dello sviluppo sostenibile (Saviano, 2014b). Certamente, incidono molto anche le attese comunemente associate all'ottenimento del riconoscimento, soprattutto quando relativo alle classificazioni del sistema UNESCO le cui prestigiose liste aprono a significative opportunità di valorizzazione e generano forti ricadute in termini di immagine e reputazione (Commissione Nazionale Italiana dell'UNESCO-IULM, 2011; Gonzales, 2013).

Le classificazioni attivate dall'UNESCO, di specifico interesse dal punto di vista dell'ambiente naturale, comprendono le liste del Patrimonio Mondiale dell'UNESCO per la conservazione di valori naturalistico-ambientali unici ed eccezionali a livello mondiale, le Riserve della Biosfera, che implicano il riconoscimento di un equilibrio tra conservazione di valori naturali, promozione della diversità naturale e culturale e modello di gestione e sviluppo sostenibile del territorio, e i Geoparchi, per i siti geologici di importanza scientifica ed estetica, nonché di valore educativo e di sviluppo territoriale (Tab. 2) (Banini, 2013; UNESCO-Division of Ecological and Earth Science, 2014).

Tab. 2: Le classificazioni UNESCO del patrimonio naturale

\begin{tabular}{|c|c|}
\hline Classificazione & Funzione \\
\hline $\begin{array}{l}\text { Patrin } \\
\text { Mon } \\
\text { dell'UN }\end{array}$ & $\begin{array}{l}\text { "Attraverso l'azione del Comitato intergovernativo per il Patrimonio } \\
\text { Mondiale, l'UNESCO incoraggia i Paesi Membri ad assicurare la protezione } \\
\text { del proprio Patrimonio naturale e culturale attraverso: } \\
\text { - l'adozione una politica generale intesa ad assegnare una funzione } \\
\text { strategica al patrimonio culturale e naturale nella vita collettiva e } \\
\text { integrando la protezione di questo patrimonio nei programmi di } \\
\text { pianificazione generale; } \\
\text { - l'istituzione sul territorio, ove non esistano ancora, di uno o più servizi } \\
\text { di protezione, conservazione e valorizzazione del patrimonio culturale e } \\
\text { naturale, dotati di personale appropriato, provvisto dei mezzi necessari } \\
\text { per adempiere i compiti che gli incombono; } \\
\text { - lo sviluppo studi e ricerche scientifiche per perfezionare i metodi } \\
\text { di intervento che permettono a uno Stato di far fronte ai pericoli che } \\
\text { minacciano il proprio patrimonio culturale o naturale; } \\
\text { - l'istituzione o lo sviluppo di centri nazionali o regionali di formazione nel } \\
\text { campo della protezione, conservazione e valorizzazione del patrimonio } \\
\text { culturale e naturale e promuovere la ricerca scientifica in questo campo"* }\end{array}$ \\
\hline $\begin{array}{c}\text { Riserve } \\
\text { della Biosfera }\end{array}$ & $\begin{array}{l}\text { "Riconosciute a seguito dell'approvazione del Programma MaB (Man and } \\
\text { the Biosphere) le Riserve della Biosfera sono "aree marine e/o terrestri che } \\
\text { gli Stati membri s'impegnano a gestire nell'ottica della conservazione delle } \\
\text { risorse e dello sviluppo sostenibile, nel pieno coinvolgimento delle comunità } \\
\text { locali. Scopo della proclamazione delle Riserve è promuovere e dimostrare } \\
\text { una relazione equilibrata fra la comunità umana e gli ecosistemi, creare siti } \\
\text { privilegiati per la ricerca, la formazione e l'educazione ambientale, oltre } \\
\text { che poli di sperimentazione di politiche mirate di sviluppo e pianificazione } \\
\text { territoriale"**. }\end{array}$ \\
\hline Geoparchi & $\begin{array}{l}\text { "I Geoparchi sono aree naturali di particolare interesse geo-minerario cui } \\
\text { l'UNESCO assegna un riconoscimento inserendoli in unapposita Rete } \\
\text { internazionale. Il punto di riferimento "europeo" della rete Unesco è la } \\
\text { "rete europea dei Geoparchi" con cui l'Unesco si coordina ed è in continuo } \\
\text { contatto"***. }\end{array}$ \\
\hline
\end{tabular}

Fonti: $\quad{ }^{*}$ http://www.unesco.it/cni/index.php/cultura/patrimonio-mondiale;

${ }^{* *}$ : www.unesco.it/cni/index.php/scienze-naturali/biosfera;

${ }^{* * *}$ http://www.unesco.it/cni/index.php/scienze-naturali/prova. 
sinergie Vol. 34, N. 99, 2016

Lappartenenza a tali classificazioni si fonda su premesse e condizioni significativamente diverse (IUCN, 2008, 2013). Spesso, tuttavia, i promotori dell'iniziativa mostrano scarsa conoscenza di tali differenze e tendono ad attribuire al riconoscimento il significato e il valore di una 'certificazione di qualità', che genera un effetto branding (Pencarelli, 2011; Tommasetti e Saccomanno, 2012; Golinelli e Sfodera, 2015). Indubbiamente, l'inserimento in una delle varie liste UNESCO assicura una forte visibilità internazionale dei siti, ma, come vedremo, se di 'certificazione' pure si trattasse, ciò che sarebbe oggetto di certificazione non sarebbe solo e tanto l'oggettiva dotazione naturalistico-ambientale, ma anche e soprattutto la soggettiva capacità di gestione di quella dotazione volta ad assicurarne la conservazione e la valorizzazione attraverso modelli di sviluppo sostenibile estesi al territorio.

Come stabilito nelle Operational Guidelines for the Implementation of the World Heritage Convention, l'iscrizione nella Lista del Patrimonio Mondiale si basa sul principio dell'eccezionale valore universale del sito (outstanding universal value). La valutazione è effettuata dall'IUCN (International Union for Conservation of Nature), organo scientifico di valutazione delle candidature, e si basa su evidenze scientifiche, sul riconoscimento internazionale e sulla comparazione effettuata con siti simili a livello globale (Colantoni, 2013). Diversamente, la candidatura a Riserva della Biosfera richiede non solo lesistenza di specifiche caratteristiche territoriali ma anche la dimostrazione di un equilibrato rapporto tra attività umane e conservazione dei valori naturali attraverso attività economiche sostenibili e tradizionali sviluppate sul territorio. Orbene, il riconoscimento è oggetto di costanti verifiche e il rischio che possa essere rimesso in discussione è elevato e dipende essenzialmente dalleffettiva implementazione di modelli ispirati allo sviluppo sostenibile sul territorio più esteso.

Quindi, ciascun programma ha specifiche finalità, la cui conoscenza e comprensione sono fondamentali non solo ai fini del riconoscimento, ma soprattutto ai fini del mantenimento dello stesso nel tempo. Adoperarsi per ottenere il riconoscimento senza portare avanti il processo in conformità con le attese, significa candidarsi a un possibile fallimento che avrebbe effetti ben più dannosi, per l'immagine e la reputazione del territorio, di quanto possa essere benefico lo stesso riconoscimento.

In sostanza, al di là delle esigenze di conservazione, dalla gestione del sistema delle aree protette ci si aspetta un significativo contributo nel favorire la concretizzazione di modelli di sviluppo sostenibile. $\grave{E}$ in tal senso che va intesa l'azione 'di sistema' ed è in tale prospettiva che, come evidenzieremo, va intesa la valorizzazione in ottica di sostenibilità, quale equilibrata ricomposizione di aspetti e interessi economici, sociali e ambientali (Faucheux et al., 1998; Patterson e Theobald, 1999; Jessop, 2000).

Tali aspetti rappresentano il cuore della nostra riflessione che si indirizza ora a evidenziare il possibile ruolo dell'impresa in questo contesto e a proporre il contributo della prospettiva dello studioso di management all'avanzamento verso modelli di sviluppo sostenibile. 


\section{Le Riserve della Biosfera del Programma MaB dell'UNESCO come modello di promozione dello sviluppo sostenibile}

L'istituzione, negli anni Settanta del secolo scorso, del Programma "Man and the Biosphere" dell'UNESCO si fondava su un già significativo impegno internazionale per la protezione dell'ambiente che anticipava quello che avrebbe poi condotto all'adozione delle Convenzioni di Rio e dei relativi principi di sviluppo sostenibile nel 1992 in occasione della nota Conferenza delle Nazioni Unite su Ambiente e Sviluppo (Colantoni, 2015).

I programmi dell'UNESCO volti a creare una rete mondiale di Riserve della Biosfera (World Reserves of Biosphere Network) - che leghi i territori locali in un sistema complessivo impegnato nella promozione dei principi e dei valori dello sviluppo sostenibile dei territori, quindi di un rapporto armonico ed equilibrato tra uomo e natura -, rappresentano una leva fondamentale per la transizione verso la sostenibilità.

Come si è detto, le Riserve della Biosfera sono luoghi istituiti, e riconosciuti ai sensi del Programma $\mathrm{MaB}$, per promuovere lo sviluppo sostenibile e conciliare la conservazione della diversità biologica e culturale con lo sviluppo economico e sociale ${ }^{2}$. Le aree protette candidate all'inserimento nel World Reserves of Biosphere Network, devono ricadere in un territorio gestito in modo integrato al fine di:

- conservare e promuovere la diversità naturale e culturale;

- funzionare come un laboratorio attivo per attività di ricerca scientifica, monitoraggio, educazione e formazione;

- fornire un modello di gestione territoriale ispirato allo sviluppo sostenibile;

- creare partnership nella rete mondiale delle Riserve della Biosfera.

La lungimirante visione dell'UNESCO individua la chiave per promuovere modelli di sviluppo sostenibile nel far convergere ricerca scientifica, politiche di conservazione della natura e sviluppo economico e sociale dei territori.

Un articolato modello di gestione del territorio, che si basa su tre funzioni fondamentali - conservazione, sviluppo e supporto logistico -, contraddistingue le attività di una Riserva $\mathrm{MaB}$, per la cui realizzazione è necessario un approccio partecipato (Di Bella e Zagarella, 2015) che assicuri:

- la risoluzione dei conflitti che comunemente si generano in tipici contesti multi-attore;

- la generazione di vantaggi diffusi a livello locale;

2 In tutto il mondo vi sono attualmente 631 Riserve della Biosfera in 119 paesi, di cui 10 in Italia. Cfr. http://www.unesco.it/cni/index.php/scienze-naturali/ biosfera; http://www.minambiente.it/pagina/le-aree-MaB-italia.

La Riserva di Biosfera di più recente istituzione in Italia è quella del Parco Nazionale della Sila, il cui iter di candidatura, avviato nel 2010, si è concluso con successo nel mese di giugno del 2014. Per un approfondito studio sulle classificazioni adottate dall'UNESCO e sul caso della Sila, si veda il Rapporto di ricerca di Sinergie, dal titolo "I territori classificati dall'UNESCO: vantaggi, potenzialità e percorsi per il Parco Nazionale della Sila". Sinergie Rapporto di ricerca N. 37, Maggio 2013. Il caso della Sila come Riserva della Biosfera riconosciuta nel Programma è trattato nellopera in corso di pubblicazione Golinelli G.M. (a cura di), Patrimonio culturale e creazione di valore. La componente naturalistica, Cedam, Padova.
Marialuisa Saviano

Il valore culturale del patrimonio naturale nella promozione dello sviluppo sostenibile 
- la valorizzazione degli stili di vita tradizionale e della conoscenza;

- il mantenimento della diversità culturale;

- gli usi locali e la conservazione della biodiversità;

- fonti alternative di reddito e di partecipazione agli utili.

La partecipazione al programma pone, quindi, come condizione necessaria ancorché non sufficiente, unampia partecipazione degli attori locali, istituzionali, imprenditoriali, sociali e dell'intera comunità. Tale partecipazione, spesso realizzata attraverso partnership pubblico-privato (Saviano et al., 2014) deve poi tradursi in consenso per esprimere le potenzialità sinergiche attese (Saviano e Magliocca, 2003; Barile e Saviano, 2012b).

Lazione di governo del territorio in ottica di sostenibilità richiede, in sostanza, che sia superata una visione mono-prospettica, se non riduzionista, di processi che, nei fatti, sono trasversali ai diversi ambiti dell'ambiente, dell'economia e della società, avendo come finalità condivisa la conservazione e valorizzazione di una diversità che integri la prospettiva naturalistico-ambientale in una più ampia prospettiva culturale.

L'interessante modello cui è affidata la capacità delle Riserve della Biosfera di evolvere da siti di conservazione ad aree di riferimento per lo sviluppo sostenibile, è basato su un efficace schema di zonizzazione del territorio che distingue tra (Fig. 2):

- core area, o area centrale, destinata alla conservazione degli ecosistemi e della biodiversità, ad attività di ricerca e monitoraggio e protetta secondo gli standard di una riserva integrale;

- buffer area, o zona tampone, esterna alla core area, destinata, da un lato, a rafforzare l'azione di protezione dell'area core, dall'altro, a una gestione delle risorse e attività locali compatibile con le finalità di protezione della Riserva;

- transition area, o area di cooperazione, non soggetta a regimi vincolistici, esterna all'area buffer, e destinata alla progettazione e sperimentazione di modelli di sviluppo sostenibile.

In sostanza, il modello promuove una visione aperta del sito attraverso un progressivo affievolimento del regime di vincolo dall'area core allarea transition, che apre a una valorizzazione della riserva come componente di più ampi sistemi territoriali di sviluppo sostenibile.

Lo spunto che emerge su cui riflettere, dunque, è nel guardare alla Riserva senza 'riserve'. Nel gioco di parole, si intende evidenziare la necessità di una visione aperta, che faccia della condizione di armonico rapporto tra uomo e natura un modello di riferimento per la promozione dello sviluppo sostenibile. I paragrafi che seguono svilupperanno tale proposta interpretativa, evidenziando il ruolo dell'impresa e dello studioso di management nel processo di transizione verso la sostenibilità promosso a livello globale. Processo che pone come questione chiave l'integrazione delle dimensioni ambientali, sociali ed economiche in un framework unitario di sviluppo sostenibile. 
Fig. 2: Il modello di zonizzazione delle Riserve della Biosfera MaB

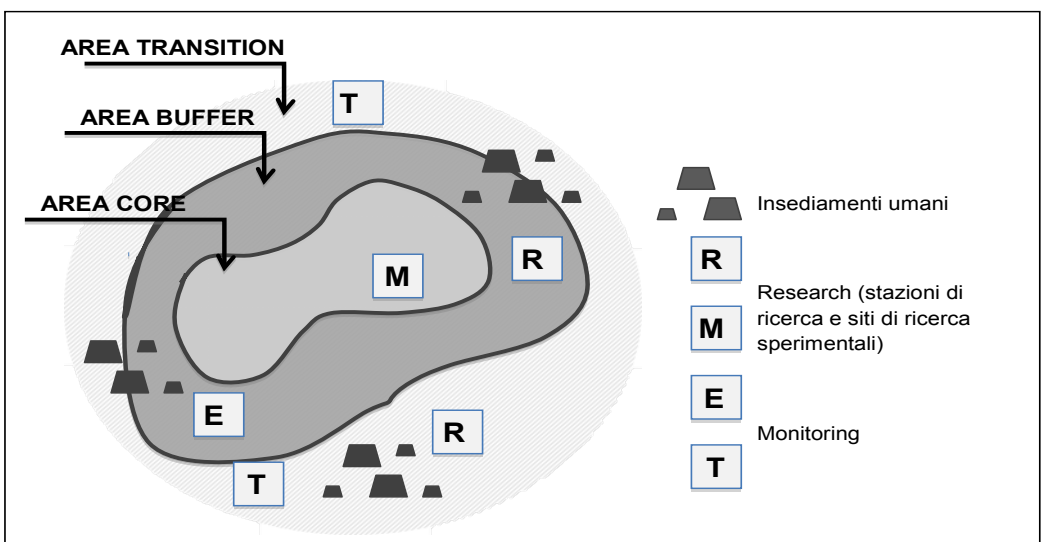

Marialuisa Saviano Il valore culturale del patrimonio naturale nella promozione dello sviluppo sostenibile

Fonte: Adattamento da UNESCO (2010)

Il più autorevole riferimento in tema di promozione dello sviluppo sostenibile, come si è detto, sono le organizzazioni delle Nazioni Unite, il cui schema di azione più generale è attualmente riconducibile all'impianto dei Sustainable Development Goals (SDGs), l'insieme di obiettivi di sviluppo sostenibile da condividere a livello globale per unazione sinergica che impatti a livello sociale, ambientale ed economico. Tale set di obiettivi, in corso di definizione, sostituirà l'impianto dei Millennium Development Goals, la cui agenda si chiude nel 2015. E attualmente in corso il Summit mondiale sullo sviluppo sostenibile (United Nations Sustainable Development Summit 2015) per l'adozione dei nuovi SDGs (Fig. 3) che condurrà alla definizione dell'agenda 2030 per lo sviluppo sostenibile (https://sustainabledevelopment. un.org/). Lo schema si basa sull'adesione dei diversi paesi a un'agenda globale definita sulla base di 17 obiettivi articolati in 169 target. Rispetto a tali obiettivi, ciascun Paese, tenendo conto delle specificità locali, definirà una propria agenda dello sviluppo sostenibile identificando priorità, strategie e modelli di azione e contribuendo al risultato globale.

Lesperienza dei Millennium Development Goals presenta risultati nel complesso soddisfacenti rispetto a diversi target. Quello che emerge, tuttavia, è la mancanza di un framework che faccia convergere sinergicamente le strategie indirizzate al raggiungimento dei singoli obiettivi integrando efficacemente le dimensioni economica, sociale e ambientale dello sviluppo sostenibile. È proprio tale esigenza che indirizza a ricondurre gli sforzi per affrontare le sfide globali del pianeta al framework generale dello sviluppo sostenibile. Si riconosce, in sostanza, la necessità di un'azione sistemica per lo sviluppo (Golinelli et al., 2015). Il problema è che le linee di azione, favorite da un impianto definito 'per obiettivi', tendono a seguire una logica 'tematica' o 'settoriale' agendo ora sul piano sociale, per esempio attraverso strategie di inclusione, ora sul piano ambientale, per esempio attraverso strategie di conservazione della biodiversità, ora sul piano economico, per esempio promuovendo il consumo responsabile. Tali azioni, tuttavia, si intrecciando generando interazioni, spesso imprevedibili, tra le dimensioni ambientali, sociali ed economiche e ponendo numerosi trade- 
sinergie Vol. 34, N. 99, 2016

off nelle scelte strategiche, di cui tener conto (Barile et al., 2015a). Ecco perché è necessario che i modelli di azione adottati contemplino la natura sistemica e unitaria delle dinamiche dello sviluppo e agiscano non solo considerando le interazioni tra le diverse dimensioni, ma anche facendo leva su di esse, attraverso un'azione cross-sectoral (Seitanidi e Crane, 2013) come esemplificato attraverso il caso del modello MaB. I modelli di azione per lo sviluppo sostenibile generalmente adottati, scontano, in sostanza, la parzialità della prospettiva, nonché la specificità degli interessi, dei soggetti che li adottano, siano essi istituzionali, sociali o imprenditoriali.

Fig. 3: L'impianto degli obiettivi di sviluppo sostenibile post-2015 (Sustainable Development Goals)

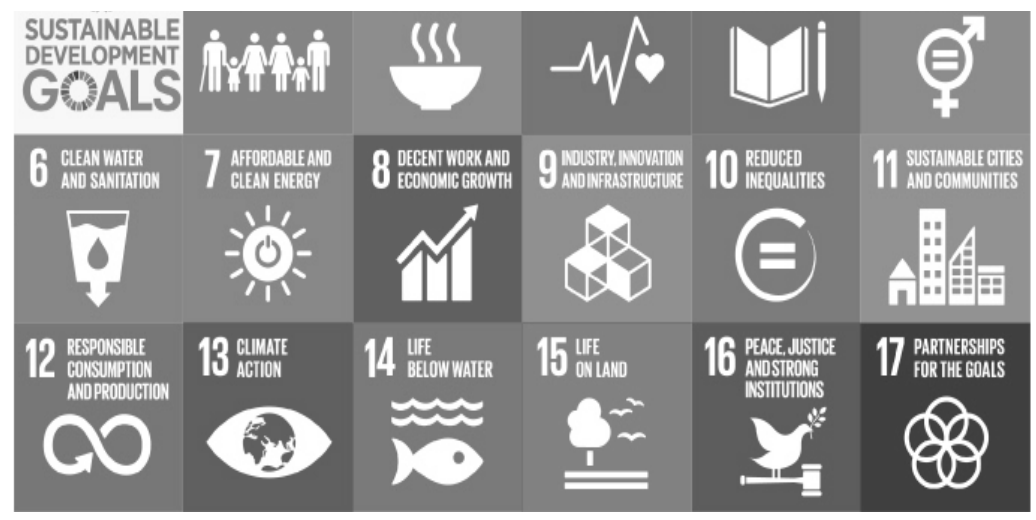

Fonte: https://sustainabledevelopment.un.org/

Esiste un'ampia varietà di approcci alla promozione dello sviluppo sostenibile, che esprimono altrettanto differenziate visioni ( $\mathrm{O}$ “Riordan, 1989), spesso disallineate (Marcuse, 1998, pag. 104): "In many cases the linking of environmental and social concerns is based on a moral (Blowers, 1993) or sympathetic outlook rather than seeing the two as materially and socially related and inseparable". (Hopwood et al., 2005, p. 10). La rappresentazione riportata in Fig. 4 propone una mappatura delle diverse visioni che integra la prospettiva ambientale e quella socioeconomica. Larea centrale ombreggiata della mappa indica la gamma di opinioni all'interno del dibattito sullo sviluppo sostenibile, che combinano problemi socio-economici e ambientali. Le visioni esterne all'area ombreggiata privilegiano, invece, la prospettiva ambientale o quella socio-economica. La mappa indica anche tre ampie visioni sulla natura dei cambiamenti nelle strutture politiche ed economiche della società e nelle relazioni uomo-ambiente necessari per raggiungere uno sviluppo sostenibile: quella della possibilità di agire senza significativi cambiamenti (Status quo); quella che ritiene necessario un approccio di riforma ma non di radicale trasformazione (Reform); quella, infine, che ritiene necessario un approccio di profonda trasformazione della società e/o delle relazioni uomo-ambiente (Transformation).

Lelemento cardine di un possibile modello generale di riferimento cui ispirarsi dovrebbe, quindi, fondarsi sull'integrazione delle dimensioni 
sociale, ambientale ed economica dello sviluppo sostenibile. Alla luce di tale assunto, ritornando al contesto del patrimonio naturale, si procede di seguito ad evidenziare come unampia prospettiva culturale possa rappresentare Marialuisa Saviano Il valore culturale del patrimonio naturale una unificante chiave di valorizzazione in ottica di sostenibilità agevolando l'integrazione delle diverse prospettive.

Fig. 4: Una mappatura degli approcci allo sviluppo sostenibile

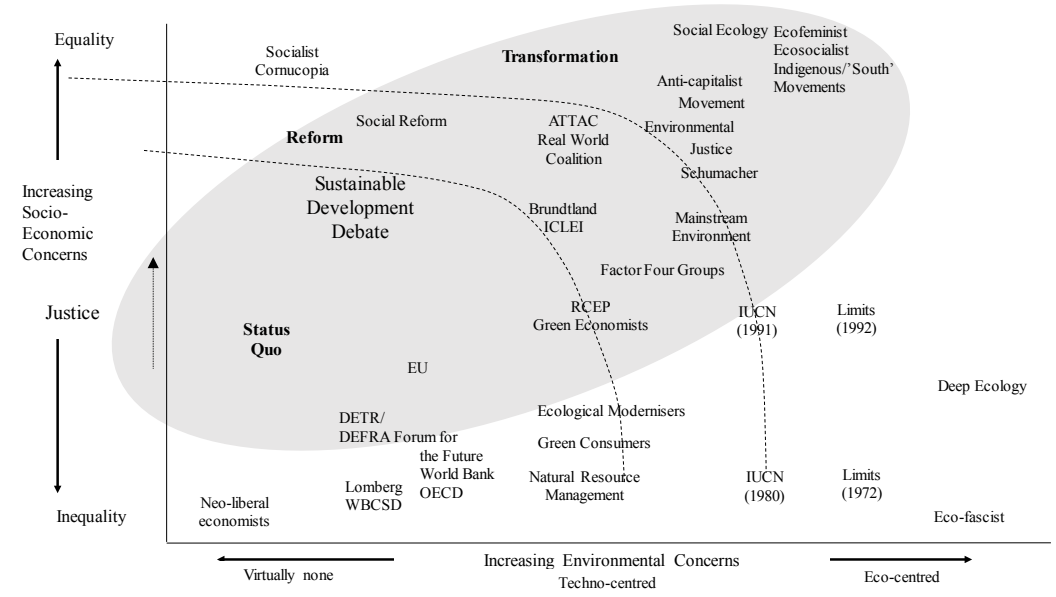

Fonte: Hopwood et al., p. 11.

\section{Verso un modello di valorizzazione culturale del patrimonio naturale nella prospettiva dello sviluppo sostenibile}

Lapproccio di gestione delle aree protette ispirato a una logica evoluta di apertura e di inclusività dei siti del patrimonio naturale nel più ampio territorio locale, si distingue da quello prevalente nei fatti, dove sembra ancora dominare una logica di protezione dell'area dall'interazione con l'ambiente circostante, tipica del regime vincolistico e della funzione di tutela (Barile e Saviano, 2012a, 2014a), che genera isolamento e chiusura relazionale (Barile et al., 2015b).

Il modello di area protetta come Riserva della Biosfera indirizza verso una piena integrazione dell'area nel territorio e unottica condivisa di sviluppo sostenibile. La zonizzazione adottata nel modello $\mathrm{MaB}$ afferma con chiarezza una visione aperta dell'area protetta. Le tre zone core, buffer e transition ambiscono a comporre un sistema territoriale vitale (Golinelli, 2002; Barile e Golinelli, 2008) finalizzato a diffondere sul territorio più ampio di riferimento un modello di sviluppo sostenibile, il cui cuore è un armonico rapporto tra uomo e natura (Spadafora, 2013).

In tal senso, una progressiva estensione dell'area sarebbe prova di una sempre più ampia condivisione del paradigma di sviluppo adottato. Affinché ciò si realizzi, occorre, tuttavia:

- unadeguata comprensione delle finalità ultime del programma;

- un elevato coinvolgimento degli stakeholder e della comunità locale; 
- il superamento della dominante visione di protezione e tutela a vantaggio di un'ottica di valorizzazione;

- una visione ampia del concetto di valore.

I comuni motivi di fallimento della candidatura, oltre a una sopravvalutazione dei valori naturali, a un regime di protezione inadatto, o a un'errata zonizzazione, sono più spesso dovuti a un inadeguato approccio di gestione del sito in un'ottica di sostenibilità.

\subsection{La promozione di un armonico rapporto tra nomo e natura come strategia di valorizzazione culturale del patrimonio naturale}

Il guardare al patrimonio naturale come opportunità di recupero di un armonico rapporto tra uomo e natura nella sua complessa multidimensionalità, mette in luce la prospettiva culturale come chiave di valorizzazione di qualsiasi forma di patrimonio.

Qui emerge il possibile contributo dell'economista d'impresa, studioso di management, nella sua capacità di riconoscere la rilevanza di unazione di governo che guardi all'area protetta quale componente di un più ampio sistema territoriale nel quale implementare modelli di sviluppo sostenibile. Si tratta di cambiare prospettiva e volgere lo sguardo dall'interno all'esterno del sito, cogliendo le sollecitazioni del progressivo allentamento del vincolo del modello $\mathrm{MaB}$ e promuovendo un complesso articolato di attività di ricerca scientifica, istruzione e formazione, nonché relazionali e di produzione economica, che, sollecitando unampia partecipazione, favoriscano la progressiva socializzazione del modello di sviluppo sostenibile sperimentato e implementato. Il collegamento in rete con altri sistemi territoriali dovrebbe poi favorire la progressiva estensione delle aree in un'ideale propensione al recupero delloriginario equilibrio ecosistemico a livello globale.

$\hat{E}$, dunque, necessario innanzitutto che l'ente di gestione del sito, nell'ambito del composito organo di governo del territorio, sviluppi una visione aperta guardando alla riserva non come patrimonio in sé, bensì come componente di un più ampio sistema territoriale dal quale possano emergere opportunità di valorizzazione (Barile e Golinelli, 2008) e nel quale la componente di dotazione sviluppi tutta la sua multi-dimensionalità arricchendosi di significati in un'ampia prospettiva culturale. Si afferma, così, una concezione sublimata di valore che "deriva in misura significativa dalla dimensione immateriale, simbolica ed esperienziale” (Tamma, 2010, p. 39; Gatti et al., 2009).

Lo sviluppo di un tale approccio di gestione richiede una dotazione di capacità e competenze manageriali (Saviano e Caputo, 2012) che sono indispensabili per rendere operativa la valorizzazione. Il focus si sposta così sulle componenti sistemiche quali istituzioni, enti e imprese che formano lorgano di governo e la struttura operativa del territorio.

La valorizzazione, quindi, si fonda su un efficace approccio di governo e di gestione del sito che veda coinvolto un insieme composito di attori. Lefficacia di tale approccio si fonda, a sua volta, sulla capacità di superare i dilemmi generalmente alla base del delicato equilibrio tra valorizzazione economica e sostenibilità ambientale, tipici dei sistemi socio-ecologici 
complessi. Infatti, le interazioni che si realizzano nei molteplici ambiti Mlvalialuisa culturale dell'economia, dell'ambiente e della società danno luogo a dinamiche del patrimonio naturale sistemiche tipiche dei Socio-Ecological Systems (Ostrom, 2009). I Socio- svilupposostenibile Ecological Systems presentano una varietà e variabilità difficili da governare essendo "both complex and evolving and their management is faced with uncertainty and surprise, making it necessary to abandon the expectation to find a global steady state" (Eneko e Sigrid, 2010, p. 1716).

Dunque, un equilibrio ecosistemico complesso (Liu et al., 2007). Un equilibrio tra ambiti dell'economia, della società e dell'ambiente fortemente interrelati, rispetto ai quali l'azione di governo sconta una diversità di prospettive che genera artificiali separazioni. Economia, ambiente e società, quali risultanti del dinamico evolvere del rapporto tra uomo e natura, hanno sviluppato paradigmi e modelli di funzionamento propri, generando squilibri nelle continue modificazioni prodotte nei diversi ambiti.

Nell'illustrare il caso delle riserve $\mathrm{MaB}$, si è implicitamente affermata una visione dei siti come 'residui' di un originario ben più esteso equilibrio ecosistemico, progressivamente perduto per effetto di squilibri nell'interazione tra i vari ambiti dell'economia, della società e dell'ambiente. La configurazione teorica delle tre dimensioni della sostenibilità trova raramente concretizzazione nella realtà. Nella situazione attuale domina, come si è detto e illustrato in Fig. 1, la sfera delleconomia tanto sulla società quanto, soprattutto, sull'ambiente, e ne risulta alterata la dinamica di funzioni e ruoli di ciascuna componente a partire dall'impresa stessa che cessa di essere motore di sviluppo e benessere diffusi in quanto imbrigliata in una miope logica speculativa e individualistica. Salta il funzionamento 'sistemico' dell'armonica interazione delle parti in un tutto unitario e si genera un anarchico 'si salvi chi può'.

La chiave per il recupero di un armonico equilibrio è in un cambiamento che è prima di tutto culturale e che può essere favorito recuperando, grazie all'adozione di una prospettiva sistemica, una visione olistica dei tre ambiti, che sfuma confini e separazioni, ricomponendo l'unitarietà del tutto, attraverso unarmonica relazionalità (consonanza) (Golinelli, 2011; Barile, 2009, Barile e Saviano, 2012b; Gatti e Esposito De Falco, 2012) e l'integrazione delle prospettive economica, sociale ed ambientale in un'ampia ottica di sostenibilità (Barile et al., 2013).

Il modello cui ispirarsi trova naturale espressione in uno sviluppo sostenibile e inclusivo e indirizzi l'organo di governo delle organizzazioni impegnate nelle diverse attività socio-economiche del territorio, a ricomporre trade-off apparentemente irriducibili, riconducendo le esigenze di efficienza, efficacia e sostenibilità ambientale ad un'ottica unitaria di sostenibilità. Ladozione dell'Approccio Sistemico Vitale (ASV) (Golinelli, 2000, 2005, 2011; Barile 2000, 2008, 2009) ha indirizzato a riflettere a fondo su tali problematiche (Golinelli e Volpe, 2012) facendo approdare al riconoscimento della sostenibilità quale chiave di volta per la ricomposizione in un quadro unitario delle diverse ottiche che indirizzano le decisioni delle organizzazioni tra efficienza economico-strutturale, efficacia sistemica ed equilibrio ecosistemico (Saviano et al., 2010; Barile et al., 2012).

Si perviene così a un possibile schema di sintesi che rilegge il modello di sistema territoriale in ottica di sostenibilità integrando le prospettive economica, sociale e ambientale (Fig. 5). 
sinergie

Vol. 34, N. 99, 2016
Fig. 5: Ampliamento di prospettiva e ottica di sostenibilità nel governo del sistema territoriale

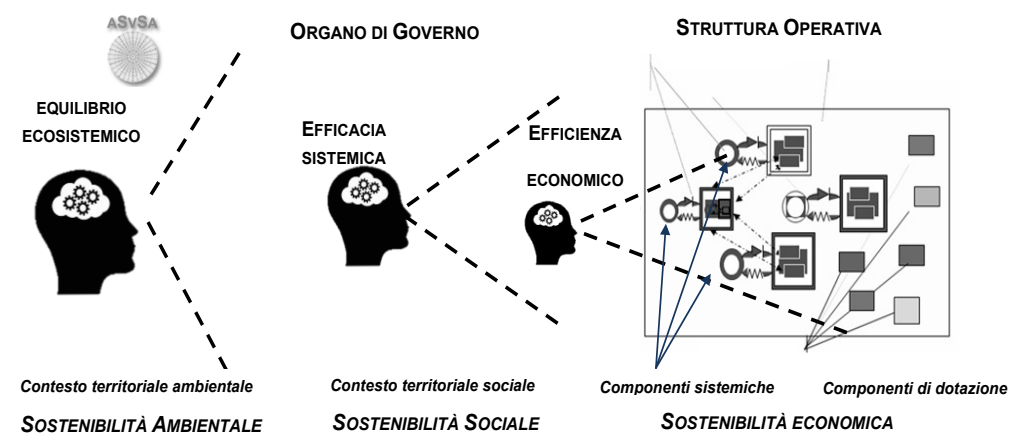

Fonte: Elaborazione da Barile e Saviano, 2012a e 2014a., www.asvsa.org

La rappresentazione proposta evidenzia come il concetto di 'sostenibilità' sia ben noto all'organo di governo quando riscontra le difficoltà economiche e finanziarie che rendono insostenibile la gestione della struttura operativa. Riconoscendo le necessità di un'efficiente gestione della struttura operativa e di un efficace governo dei rapporti inter-sistemici, lo schema interpretativo proposto rilegge gli obiettivi di efficienza economico-strutturale locale come esigenze di sostenibilità economica, quelli di efficacia sistemica di contesto come esigenze di sostenibilità sociale e quelli di equilibrio ecosistemico come esigenze di sostenibilità ambientale, riconducendo così le tre prospettive a unottica unitaria di sostenibilità.

Dunque, unazione di governo orientata alla valorizzazione in ottica di sostenibilità, così come esemplificato nel caso del modello MaB, deve essere capace di catalizzare e conciliare unampia varietà di interessi di cui sono portatori diversi soggetti, generando una sempre più estesa consonanza $d i$ contesto (Barile e Calabrese, 2011). In tal modo, le condizioni di equilibrio ecosistemico interno alla 'riserva' possono estendersi progressivamente all'esterno della stessa.

Rispetto a tale rappresentazione ideale, occorre essere consapevoli che, fino a quando la sostenibilità non sarà diffusamente riconosciuta come valore, sarà difficile che si passi "dalla filosofia alloperatività" (Golinelli e Volpe, 2012). In tale contesto, l'impresa, illuminata da una visione evoluta del proprio ruolo, lungi dal vedersi vincolata e limitata, deve manifestare capacità non già di adeguamento o trasformazione, ma di più radicale ripensamento delle sue logiche di azione, dimostrando così di essere un attore determinante nella transizione verso modelli di sviluppo sostenibile.

\subsection{Il ruolo dell'impresa e del management nella promozione dello sviluppo sostenibile}

Il ruolo dell'impresa in un sistema territoriale - locale e idealmente globale -, orientato allo sviluppo sostenibile è decisivo. L'impresa è l'attore 'realizzativo' dello sviluppo che, convogliando le risorse dell'ambiente sulla base di istanze recepite dal contesto sociale, nel rispetto di vincoli e regole 
condivisi, determina le concrete traiettorie evolutive, impattando sui livelli complessivi di sostenibilità nei diversi ambiti (Saviano, 2014a).

In tale contesto, è compito dello studioso di management guidare il rinnovamento dell'impresa, 'aprendo la mente' dell'imprenditore ed evidenziandogli modi nuovi di concepire il proprio ruolo nel contesto.

La rappresentazione di sintesi sopra proposta non pone le legittime e ovvie necessità di un equilibrio economico, finanziario e anche monetario del sistema (Metallo e Cuomo, 2008), in contrasto con le attese che maturano a livello sociale e nella più ampia prospettiva dell'ambiente. Il decisore comprende che le condizioni di performance di una tradizionale ottica di 'profitto' passano per un complesso di attività negoziali e relazionali di contesto che si sviluppano con una varietà di attori - esterni alla struttura operativa, ma interni al sistema. Le condizioni di raccordo con ambiente e mercati devono derivare dalla capacità di proporre un progetto condiviso che crei nel contesto condizioni durevoli di performance (Vito, 1986; Paniccia et al., 2010; Minguzzi e Solima, 2012) basate sull'integrazione delle risorse in un'ottica di co-creazione di valore (Prahalad e Ramaswamy, 2004; Vargo e Lusch, 2006; Payne et al., 2008; Porter e Kramer, 2011; Barile e Saviano, 2014a; Polese et al., 2014). Un tale approccio genera nel territorio rilevanti meccanismi di funzionamento ecosistemico (Vargo, 2006; Vargo e Lusch, 2010; Chandler e Vargo, 2011; Wieland et al., 2012) che incrementano le potenzialità di creazione di valore.

L'impresa, quale componente sistemica per eccellenza, ossia attore dotato di capacità cognitive, decisionali, strategiche e relazionali, quindi di risorse di conoscenza (Conner e Prahalad, 1996; Kandampully, 2002; Vargo et al., 2008; Montella, 2008), deve guardare alle componenti di dotazione del territorio (il sito, nel nostro caso) intercettando nel contesto opportunità di valorizzazione, attraverso una dinamica combinazione e ricombinazione di risorse, capacità e competenze (Carrus e Melis, 2006; Siano et al., 2006; Barile et al., 2014).

Affinché il potenziale delle componenti di dotazione di un territorio possa esprimersi pienamente è, dunque, necessaria unazione di valorizzazione che individui creativamente funzionalità e ruoli capaci di innestarsi armonicamente in sistemi più ampi di azione facendo leva sulla sostenibilità anche come opportunità di differenziazione competitiva. Per questo, è importante che, come modellizzato nel programma MaB, si realizzino attività di ricerca scientifica e di istruzione e formazione, per uno sviluppo socio-economico basato, innanzitutto, sulla inesauribile risorsa di conoscenza (Rullani, 2005), assegnando a scuola, università e mondo della ricerca la responsabilità di rendere la sostenibilità un paradigma generale di riferimento che indirizzi lo sviluppo di innovazioni non solo sostenibili in se stesse ma capaci di promuovere la sostenibilità (Barile et al., 2012).

Dall'impresa il cui ruolo sia così concepito, non ci si deve, pertanto, attendere un atteggiamento di 'risposta' alla sfida della sostenibilità. L'impresa non deve 'rispondere', deve 'chiamare' a rispondere alla sfida della sostenibilità, assumendo una rinnovata centralità. Questo è il suo ruolo adesso. È un profondo ripensamento di se stessa, che l'impresa dovrà in ogni caso affrontare, perché, per come è stata tradizionalmente concepita e tuttora funziona, non potrà 'sostenere' l'impegno per la sostenibilità.
Marialuisa Saviano

Il valore culturale del patrimonio naturale nella promozione dello

sviluppo sostenibile 
sinergie Vol. 34, N. 99, 2016

Non si tratta, dunque, solo di rispetto per l'ambiente, di responsabilità sociale, né di filantropia o mecenatismo. Si tratta di un ripensamento più profondo del ruolo dell'impresa, che va oltre gli assunti della Stakeholder Theory (Freeman, 1984) e della Corporate Social Responsibility (CSR) (Carrol, 1979). È il più ampio set di stakeholder di riferimento, sia pure in ragione di una differenziata rilevanza, che diviene strategico per la sostenibilità stessa dellimpresa: "the sustainability of the company depends on the sustainability of its stakeholder relationships" (Tencati e Zsolnai, 2009, p. 374). Perdono di efficacia tanto i sistemi 'impositivi' quanto i sistemi 'motivanti' all'impegno per la sostenibilità. Valga l'esempio dell'Emission Trading Scheme (ETS) (Vagnani, 2005; Convery e Redmond, 2007), sistema per la riduzione delle emissioni certamente efficace nel breve, ma molto debole e lento, se non controproducente, nel favorire il cambiamento culturale necessario alla transizione verso la sostenibilità. Come questo, altri sistemi attualmente adottati tradiscono una concezione dell'impresa, che si vorrebbe superare, in cui ancora dominano logiche opportunistiche e speculative.

Sia chiaro che alla rappresentazione consapevolmente idealistica che si sta delineando, e che potrebbe comprensibilmente risultare anche ingenua, non sfugge il fatto che lauspicato cambiamento paradigmatico forse non giungerà a compimento. Ma questa consapevolezza profonda non sottrae lo studioso di management alla responsabilità di non rendersi 'complice' di una rassegnata inesorabilità dei 'mali' della società. Ed è proprio allo studioso di economia d'impresa che spetta il compito di coniugare lo sviluppo di una conoscenza tecnica e tecnologica con una conoscenza di portata ben più ampia che incida sulla formazione dei valori e del metodo oltre che delle competenze nel rispondere alle istanze dinamicamente emergenti dal contesto (Caselli, 2003; Barile et al., 2014; Massaroni et al., 2014; Saviano, 2014b; Simone et al., 2014).

Si tratta, quindi, di un cambiamento che sposta l'attenzione su valori e schemi generali che indirizzano scelte e comportamenti di una comunità. In questo spostamento di focus si coglie pienamente la centralità della dimensione culturale nella valorizzazione del patrimonio.

\section{Riflessioni conclusive}

Negli studi sulla sostenibilità e sullo sviluppo sostenibile nella prospettiva dell'economia d'impresa, sulla linea ormai consolidata di un approccio ispirato alla Stakekolder Theory, prevale, attualmente, lottica della Corporate Social Responsibility (Carroll, 1979, 1991; Caselli, 2005; Habisch et al., 2005; Perrini e Tencati, 2008; Eisingerich e Ghardwaj, 2011) e, in tema di sviluppo sostenibile, si insiste sulla necessità di adottare sistemi di accountability e di reporting che 'diano conto' dell'impatto dell'impresa non solo sulleconomia, ma anche sulla società e, soprattutto, sullambiente (Gray et al., 1988; Tenuta, 2009; Siano, 2012). Questa visione, in sintesi, afferma che l'impresa non deve più considerarsi 'affare' solo della proprietà o del management, ma deve 'dar conto' a un più ampio insieme di portatori di legittimi interessi (Matten e Moon, 2008). Implicitamente, 
tuttavia, questa visione afferma anche un 'decadimento' nella capacità dell'impresa di generare benessere e sviluppo diffusi, ruolo che vedrebbe non solo legittimata ma anche riaffermata la sua funzione, e accettata una forma di 'dominanza'. L'impresa è vista come 'controparte' piuttosto che partner, secondo una visione transazionale dei rapporti con ambiente e mercati, ormai superata nella teoria, anche se non certamente nella pratica, che ne fa decadere funzione e ruolo.

Nel modello della consonanza proposto dall'ASV si afferma una rinnovata visione dell'impresa e della competitività, che guarda al futuro (World Commission on Environment and Development, 1987; Hamel e Prahalad, 2013), come capacità di co-evolvere e di co-creare il valore con unampia varietà di attori. L'economia d'impresa coglie tale esigenza e teorizza modelli che orientano il decisore nelle scelte di governo, riconoscendo la consonanza quale naturale propensione nella ricerca di condizioni di vitale sopravvivenza nel contesto (Maggioni, 2010). Ghoshal (2005) sintetizza in modo esemplare tale necessità evidenziando la necessità che le teorie di management superino i limiti dei loro modi causali e funzionali di spiegare i fenomeni che escludono l'etica e la moralità: "In other words, a precondition for making business studies a science as well as a consequence of the resulting belief in determinism has been the explicit denial of any role of moral or ethical considerations in the practice of management" (pag. 79). Il concetto di vitalità basato sulla consonanza magnifica e riorienta la visione competitiva delle interazioni intra e inter-organizzative, agevolando la risoluzione di molti dilemmi decisionali che rendono ardua l'azione di governo in ottica di sostenibilità.

Dunque, uno sviluppo armonico (Baccarani e Golinelli, 2011) basato sulla creazione di sinergie, in cui ciascuno ha un ruolo, sapendo di doversi innestare armonicamente in un sistema di vincoli e regole non già da subire come limitanti la libertà di condotta e le relative performance, bensì da cogliere attraverso la generazione di un funzionamento ecosistemico.

Guardando al caso delle Riserve della Biosfera MaB quale metafora di una più generale rappresentazione, si coglie il nodo della riflessione proposta: anche l'impresa deve uscire dalla logica di 'proteggere' se stessa da regimi vincolistici subiti come 'controparte' di rapporti non pienamente dominati, rendendosi invece protagonista del cambiamento, progettista e attivatore di una radicale trasformazione basata sulla creazione di ampi spazi di consonanza e sulla capacità sistemica di co-evolvere armonicamente con ambiente e società.

In tale scenario, la sostenibilità diventa l'opportunità che si presenta all'impresa di ripensare se stessa, uscendo dalla condizione di disorientamento e dalla crisi di identità in cui è caduta e tracciando un nuovo percorso da seguire. In questa prospettiva, la sostenibilità stessa risulterebbe ripensata ergendosi da risultante dell'intreccio di tre prospettive della 'sostenibilità' (economica, sociale e ambientale), a paradigma generale di riferimento che definisce principi e regole comuni di condotta cui orientarsi.

La riflessione proposta auspica, quindi, un cambiamento culturale che investa l'impresa così come le istituzioni e la collettività nei molteplici rapporti tra economia, ambiente e società, di cui la ricerca di management deve farsi interprete (Pogutz e Winn, 2013). Un cambiamento culturale
Marialuisa Saviano Il valore culturale del patrimonio naturale nella promozione dello sviluppo sostenibile 
sinergie Vol. 34, N. 99, 2016

decisivo per uneffettiva transizione verso la sostenibilità, che lo studioso di management deve guidare, favorendo l'emergere di un nuovo paradigma (Sen, 1986) in un contesto di superficiale condivisione e profondo disinteresse.

\section{Bibliografia}

AA.VV. (2003), AP - Il sistema nazionale delle aree protette nel quadro europeo: classificazione, pianificazione e gestione, Convenzione tra il Ministero dell'Ambiente, Servizio Conservazione della Natura e il CED-PPN (Centro Europeo di Documentazione sulla Pianificazione dei Parchi Naturali) Politecnico e Università di Torino, Alinea Editrice.

ADEBOWALE M. (2002), “Towards a socially inclusive sustainable development research agenda”, in Eames M., Adebowale M., Sustainable development and social inclusion. Towards an integrated approach to research, York Publishing Services Ltd, York, pp. 4-16.

ARRIGO E. (2008), Responsabilità aziendale in economia di scarsità. Il caso Olivetti, Giappichelli, Torino.

BACCARANI C., GOLINELLI G.M. (2011), "Per una rivisitazione delle relazioni tra impresa e territorio", Sinergie, n. 84, pp. VII- XIII.

BANINI S. (2013), "Le classificazioni UNESCO: uno sguardo d'insieme", Sinergie Rapporto di ricerca, n. 37, pp. 13-44.

BARILE S. (2000), Contributi sul pensiero sistemico in economia d'impresa, Arnia, Salerno.

BARILE S. (2008), L'impresa come sistema, Giappichelli, Torino.

BARILE S. (2009), Management Sistemico Vitale, Giappichelli, Torino.

BARILE S. (2012), "Verso una novata ipotesi di rappresentazione del concetto di bene culturale", in Golinelli G.M. (a cura di), Patrimonio culturale e creazione di valore. Verso nuovi percorsi, Cedam, Padova, pp. 71-96

BARILE S. (2014), "Towards a Novel Conception of Bene Culturale”, in Golinelli G.M. (Ed.), Cultural Heritage and Value Creation, Springer International Publishing, New York, pp. 53-70.

BARILE S., GOLINELLI C.M. (2008), "Modalità e limiti dell'azione di governo del territorio in ottica sistemica", in Barile S. (a cura di), L'impresa come sistema. Contributi sull'Approccio Sistemico Vitale (ASV), Giappichelli, Torino, pp. 243-268.

BARILE S., CALABRESE M. (2011), "Business design e consonanza di contesto”, Sinergie rapporti di ricerca, n. 11, pp. 1-26.

BARILE S., MONTELLA M., SAVIANO M. (2011), "Enhancement, Viability and Value of Cultural Heritage. Towards a Service-Based Systems Approach”, in Gummesson E., Mele C., Polese F. (eds), The 2011 Naples Forum on Service. Service-Dominant Logic, Network \& Systems Theory and Service Science: integrating three perspectives for a new service agenda, Giannini Editore, Napoli.

BARILE S., SAVIANO M. (2012a). "Dalla Gestione del Patrimonio di Beni Culturali al Governo del Sistema dei Beni Culturali", in Golinelli G.M., (a cura di), Patrimonio culturale e creazione di valore, Verso nuovi percorsi, Cedam, Padova, pp. 97-148. 
BARILE S., SAVIANO M. (2012b), "Oltre la partnership: un cambiamento di prospettiva", in Esposito De Falco S., Gatti C. (a cura di), La consonanza nel governo dell'impresa. Profili teorici e applicazioni, Franco Angeli, Milano, pp. 56-78.

BARILE S., SAVIANO M., IANDOLO F. (2012), "L'innovazione tra creatività e sostenibilità", in Barile S., Polese F., Saviano M. (a cura di), Immaginare l'innovazione, Giappichelli, Torino, pp. 103-140.

BARILE S., SAVIANO M., POLESE F., DI NAUTA P. (2013), "Il rapporto impresaterritorio tra efficienza locale, efficacia di contesto e sostenibilità ambientale", Sinergie, n. 90, pp. 25-49.

BARILE S., SAVIANO M. (2014a), "Resource integration and value co-creation in cultural heritage management", in Aiello L., (Ed.), Management of Cultural Products: E-Relationship Marketing and Accessibility Perspectives, IGI Global, Hershey, PA, pp. 58-82.

BARILE S., SAVIANO M. (2014b), "From the Management of Cultural Heritage to the Governance of the Cultural Heritage System", in Golinelli G.M., (Ed), Cultural Heritage and Value Creation. Towards New Pathways, Springer International Publishing, New York, pp. 71-103.

BARILE S., SAVIANO M., SIMONE C. (2014), "Service economy, knowledge and the need for T-shaped Innovators", World Wide Web, vol. 18, n. 4, pp. 11771197.

BARILE S., SAVIANO M., IANDOLO F., CAPUTO F. (2015a), "La dinamica della sostenibilità tra vortici e correnti", in XXXVII Convegno Nazionale AIDEA "Sviluppo, sostenibilità e competitività delle aziende: il contributo degli economisti aziendali”, Piacenza, 10-11-12 settembre 2015.

BARILE S., TOMMASETTI A., SAVIANO M. (2015b), "I parchi e le aree protette tra funzione di tutela e finalità di valorizzazione in ottica di sostenibilità,, in Golinelli G.M. (a cura di), Patrimonio culturale e creazione di valore. La componente naturalistica, Cedam Kluwer, Padova, pp. 67-114.

BAUER R.A. (1966), Social Indicators, The MIT Press, Cambridge.

BEBBINGTON J., UNERMAN J., O’DWYER B. (eds) (2014), Sustainability accounting and accountability, Routledge, Londra.

BLOWERS A. (1993), “The Time for Change”, in Blowers A. (ed.), Planning for a Sustainable Environment, Earthscan, London.

BONEL E., MORETTI A., RISPOLI M., TAMMA M. (2005), "I prodotti culturali in una prospettiva economico-manageriale", Economia della cultura, vol. 15, n. 4, pp. 497-512.

BONFANTI A. (2009), "Sviluppo sostenibile in azione: il ruolo delle Banche di Credito Cooperativo nella comunità locale", Mercati e Competitività, fascicolo 2, pp. 61-81.

CAFFERATA R.(1987), “Un'interpretazione dialettica del rapporto tra organizzazione e ambiente esterno", Finanza marketing e produzione, fascicolo 2, pp. 69-108.

CARRUS P.P., MELIS G. (2006), L'innovazione delle scelte strategiche in condizioni di crescente complessità. Risorse, processi e competenze per la creazione di valore nel nuovo scenario, Franco Angeli, Milano.

CARROLL A.B. (1979), "A three-dimensional conceptual model of corporate social performance ", Academy of Management Review, vol. 4, n. 4, pp. 497-505.

CARROLL A.B. (1991), "The pyramid of corporate social responsibility: Toward the moral management of organizational stakeholders", Business Horizons, vol. 34, n. 4 , pp. 39-48. Marialuisa Saviano
Il valore culturale del patrimonio naturale nella promozione dello sviluppo sostenibile 
Sinergie Vol. 34, N. 99, 2016

CARROLL A., BUCHHOLTZ A. (2014), Business and society: Ethics, sustainability, and stakeholder management, Cengage Learning, United Kingdom.

CASELLI L. (2003), "La ri-legittimazione sociale dell'impresa”, Sinergie, n. 61-62, pp. 117-131.

CASELLI L. (2005), "La responsabilità social dell'impresa tra democrazia e mercato", Sinergie, n. 67, pp. 45-53.

CHANDLER J.D., VARGO S.L. (2011), "Contextualization and value-in-context: How context frames exchange", Marketing Theory, vol. 11, n. 1, pp. 35-49.

CIASULLO M.V., TROISI O. (2013), "Sustainable value creation in SMEs: A case study”, The TQM Journal, vol. 25, n. 1, pp. 44-61.

COLANTONI M. (2013), "La comparazione su scala regionale e globale”, Sinergie, n. 37, pp. 121-153.

COLANTONI M. (2015, in corso di pubblicazione), "I punti di forza della candidatura di un parco nazionale alla rete mondiale delle riserve della biosfera UNESCO: il caso della Sila", in Golinelli G.M. (a cura di), Patrimonio culturale e creazione di valore. La componente naturalistica, Cedam, Padova.

COMMISSIONE NAZIONALE ITALIANA DELL'UNESCO-IULM (2011), Presentazione risultati ricerca "Il valore del brand UNESCO", http://www. unesco.it/_filesSTAMPA/2011/CS_BRAND_UNESCO.pdf.

CONNER K.R., PRAHALAD C.K. (1996), "A resource-based theory of the firm: Knowledge versus opportunism”, Organization science, vol. 7, n. 5, pp. 477 501.

CONVERY F.J., REDMOND L. (2007), "Market and price developments in the European Union emissions trading scheme", Review of Environmental Economics and Policy, vol. 1, n. 1, pp. 88-111.

CRANE A., PALAZZO G., SPENCE L.J., MATTEN D. (2014), "Contesting the value of "creating shared value"', California management review, vol. 56, n. 2, pp. 130-153.

DI BELLA O., ZAGARELLA A. (2015 in corso di pubblicazione), "Fare rete: il network mondiale e le Reserves Associations del Programma UNESCO "Man and the Biosphere"', in Golinelli G.M. (a cura di), Patrimonio culturale e creazione di valore. La componente naturalistica, Cedam, Padova.

DE TONI A.F., COMELLO L. (2005), Prede o ragni, Utet libreria, Torino.

EISINGERICH A.B., GHARDWAJ G. (2011), "Corporate Social Responsibility: Does Social Responsibility Help Protect a Company's Reputation?”, MIT Sloan Management Review, n. 52, p. 18.

ELKINGTON J. (1994), “Towards the suitable corporation: win-win-win business strategies for sustainable development", California management review, vol. 36, n. 2, pp. 90-100.

ELKINGTON J. (1998), Cannibals with forks: the triple bottom line of twenty first century business, Capstone, Londra.

ELKINGTON J. (2004), "Enter the triple bottom line”, in Henriques A., Richardson J., (eds), The triple bottom line: Does it all add up, Routledge, London, pp. $1-16$.

ENEKO G., SIGRID S. (2010), "Public participation for sustainability and social learning: Concepts and lessons from three case studies in Europe", Ecological Economics, vol. 69, n. 8, pp. 1712-1722. 
FAUCHEUX S., O'CONNOR M., VAN DER STRAATEN J. (1998), Sustainable development: Concepts, rationalities and strategies, Springer Netherlands.

FEDERPARCHI (2009), Loro verde d'Italia. Percorsi, esperienze e valori del sistema delle aree naturali protette, Europarc, http://www.areeprotette. provincia.tn.it/binary/pat_aree_protette/documentazione/Dossier_Oro_ Verde.1350639039.pdf.

FERRARA G. (1994), "Il rapporto impresa-ambiente: la ricerca nelle discipline economico-aziendali", Economia e diritto del terziario, vol. 6, n. 2, pp. 551562.

FERRARI S. (2013), "Introduzione”, Sinergie Rapporto di ricerca, n. 37, Maggio, pp. 9-12.

FRANCH M. (2010), "Le frontiere manageriali per la valorizzazione della cultura e dell'arte”, Sinergie, n. 82, pp. 95-107.

FREEMAN R.E. (1984), Strategic management: a stakeholder approach, Pitman, London.

FREY M., IRALDO F. (2009), Il management dellambiente e della sostenibilità oltre $i$ confini aziendali. Dalle strategie d'impresa alla governance nei sistemi produttivi territoriali, Franco Angeli, Milano.

GATTI M., BIFERALI D., VOLPE L. (2009), "Il governo dell'impresa tra profitto e creazione di valore”, Sinergie, n. 79, pp. 145-171.

GATTI C., ESPOSITO DE FALCO S. (2012), La consonanza nel governo d'impresa: profili teorici e applicazioni, Franco Angeli, Milano.

GHOSHAL S. (2005), "Bad management theories are destroying good management practices", Academy of Management learning and education, vol. 4, n. 1, pp. 75-91.

GONZALES P. (2013), "I vantaggi legati al riconoscimento di un territorio”, Sinergie Rapporto di ricerca, n. 37, pp. 45-55.

GOLINELLI C.M. (2002), Il territorio sistema vitale: verso un modello di analisi, Giappichelli, Torino.

GOLINELLI G.M. (2000), L'approccio sistemico al governo d'impresa. L'impresa sistema vitale, vol. I, Cedam, Padova.

GOLINELLI G.M. (2005), L'approccio sistemico al governo dell'impresa. L'impresa sistema vitale, vol. I, Cedam, Padova.

GOLINELLI G.M. (2011), L’approccio sistemico vitale (ASV) al governo dell'impresa. Verso la scientificazione dellazione di governo, vol. II, Cedam, Padova.

GOLINELLI G.M. (a cura di) (2012), Patrimonio culturale e creazione di valore, Verso nuovi percorsi, Cedam, Padova.

GOLINELLI G.M. (Ed.) (2014), Cultural Heritage and Value Creation. Towards New Pathways, Springer International Publishing, New York.

GOLINELLI G.M. (a cura di) (2015), Patrimonio culturale e creazione di valore. La componente naturalistica, Cedam Kluwer, Padova.

GOLINELLI G.M., VOLPE L. (2012), Consonanza, valore, sostenibilità: verso l'impresa sostenibile, Cedam, Padova.

GOLINELLI G.M., SFODERA F. (2015), "Il contributo del marketing management alla valorizzazione delle aree naturali protette: la necessità di un modello ad hoc", in Golinelli G.M. (a cura di), Patrimonio culturale e creazione di valore. La componente naturalistica, Cedam Kluwer, Padova.
Marialuisa Saviano

Il valore culturale

del patrimonio naturale

nella promozione dello

sviluppo sostenibile 
sinergie Vol. 34, N. 99, 2016

GOLINELLI G.M., BARILE S., SAVIANO M., FARIOLI F., YARIME M. (2015), "Towards a common framework for knowledge co-creation: opportunities of collaboration between Service Science and Sustainability Science", in Gummesson E., Mele C., Polese F. (Eds.) (2015), Service Dominant Logic, Network and Systems Theory and Service Science: Integrating three Perspectives for a New Service Agenda, Giannini, Napoli, pp. 1-25.

GRAY R., OWEN D., MAUNDERS K. (1988), "Corporate social reporting: emerging trends in accountability and the social contract", Accounting, Auditing and Accountability Journal, vol. 1, n. 1, pp. 6-20.

HABISCH A., JONKER J., WAGNER M., SCHMIDPETER R. (2005), Corporate Social Responsibility Across Europe, Springer, New York.

HAMEL G., PRAHALAD C.K. (2013), Competing for the Future, Harvard Business Press, Boston MA.

HARDIN G. (1968), “The tragedy of the commons", Science, vol. 162, n. 3859, pp. 1243-1248.

HOPWOOD B., MELLOR M., O’BRIEN G. (2005), “Sustainable development: mapping different approaches", Sustainable development, Wiley Online Library, London. http://nrl.northumbria.ac.uk/9387/1/Mapping Sustainable_Development.pdf (settembre 2015).

IUCN (2006), The Future of Sustainability Re-thinking Environment and Development in the Twenty-first Century, Report of the IUCN Renowned Thinkers Meeting, 29-31 January 2006.

IUCN (2008), Guidelines for Applying Protected Area Management Categories, World Commission on protected area.

IUCN (2013), Linking Landscapes Exploring the relationships between World Heritage cultural landscapes and IUCN protected areas, IUCN, Gland, Switzerland.

JESSOP B. (2000), "Globalisation, entrepreneurial cities and the social economy", in Hamel M., Lustiger-Thaler M. (eds.), Urban movements in a globalising world, Routledge, London, pp. 81-100.

LIU J., DIETZ T., CARPENTER S.R., ALBERTI M., FOLKE C., MORAN E., TAYLOR W.W. (2007), "Complexity of coupled human and natural systems”, Science, vol. 317, n. 5844, pp. 1513-1516.

MAGGIONI V. (2010), "Una rimeditazione dei rapporti tra profitto e valore alla ricerca di una consonanza intersistemica”, Sinergie, n. 81, pp. 117-134.

MAIZZA A. (2013), "Impresa, territorio, competitività: riflessioni e prospettive di ricerca”, Sinergie, n. 90, pp. 11-21.

MARCUSE P. (1998), "Sustainability is not enough", Environment and Urbanization, vol. 10, n. 2, pp. 103-112.

MASSARONI E., ROSSI S. (2007), "Utilizzo e distribuzione delle risorse naturali: verso l'impresa eco-efficiente", Economia e Diritto del Terziario, vol. 19, n. 1, pp. 1000-1032.

MASSARONI E., ESPOSITO DE FALCO S., SANCETTA G., COZZOLINO A., BILOTTA A., CARRUBBO L. (2014), "Alla ricerca di un possibile principio evolutivo della teoria e della pratica d'impresa. Dinamiche di sviluppo delle modalità di produzione industriale", in XXVI Convegno annuale di Sinergie "Manifattura: quale futuro?", 13-14 Novembre, Cassino.

MATTEN D., MOON, J. (2008), “'Implicit” and "explicit” CSR: a conceptual framework for a comparative understanding of corporate social responsibility", Academy of management Review, vol. 333, n. 2, pp. 404-424. 
MEADOWS D.H., MEADOWS D.L., RANDERS J. (1972), The limits to growth, Ml Marialuisa Saviano Universe Books, New York.

METALLO G., CUOMO M.T. (2008). "Verso l'impiego di modelli economico-- $\begin{gathered}\text { nella promozione } \\ \text { sviluppo sostenibile }\end{gathered}$ finanziari per la valutazione e la valorizzazione del Patrimonio Artisticoculturale. L'esperienza dell'ambito di Nuceria Alfaterna", Sinergie, n. 77, pp. 187-209.

MINGUZZI A., SOLIMA L. (2012), "Relazioni virtuose tra patrimonio culturale, turismo e industrie creative a supporto dei processi di sviluppo territoriale", in XXIV Convegno annuale di Sinergie 2012 "Il territorio come giacimento di vitalità per l'impresa”, Università degli Studi del Salento, 18-19 ottobre, Lecce.

MONTELLA M. (2008), "Conoscenza e informazione del cultural heritage come spazio d'impresa", Sinergie, n. 76, pp. 91-11.

MONTELLA M. (2009), Valore e valorizzazione del patrimonio culturale storico, Mondadori Electa, Milano.

MONTELLA M. (2012), "Valore culturale", in Golinelli G.M. (a cura di), Patrimonio culturale e creazione di valore, Verso nuovi percorsi, Cedam, Padova, pp. 3-70.

MONTELLA M. (2014), "Cultural Value", in Golinelli G.M. (Ed.), Cultural Heritage and Value Creation. Towards New Pathways, Springer, New York, pp. 1-51.

MONTELLA M. (2015), "Identità e missione dei beni ambientali e delle aree protette”, in Golinelli G.M. (a cura di), Patrimonio culturale e creazione di valore. La componente naturalistica, Cedam Kluwer, Padova.

O“RIORDAN T. (1989), “The Challenge for Environmentalism”, in Peet R., Thrift N. (eds.), New Models in Geography, Unwin Hyman, London.

OSTROM E. (1999), "Coping with tragedies of the commons", Annual review of political science, vol. 2, n.1, pp. 493-535.

OSTROM E. (2009), "A General Framework for Analyzing Sustainability of SocialEcological Systems", Science, vol. 325, pp. 419-422.

PANICCIA P., SILVESTRELLI P., VALERI M. (a cura di) (2010), Economia e management delle attività turistiche e culturali. Destinazione, impresa, esperienza contributi di ricerca, Giappichelli, Torino.

PAYNE A.F., STORBACKA K., FROW P. (2008), "Managing the co-creation of value", Journal of the academy of marketing science, vol. 36, n. 1, pp. 83-96.

PATTERSON A., THEOBALD K.S. (1999). "Emerging contradictions: sustainable development and the new local governance", in Buckingham-Hatfield S., Percy S. (eds.), Constructing Local Environmental Agendas: People, Places and Participation, Routledge, London, pp. 156-171.

PELS J., BARILE S., SAVIANO M., POLESE F., CARRUBBO L. (2014), “The contribution of VSA and SDL perspectives to strategic thinking in emerging economies", Managing Service Quality, vol. 24, n. 6, pp. 565-591.

PENCARELLI T. (2011), "Il branding territoriale e dei beni culturali", in Montella M., Cerquetti M. (a cura di), Economia, cultura, territorio, eum, Macerata, pp. 27-43.

PENCARELLI T. (2015, a cura di), Comunicare le destinazioni balneari. Il ruolo delle Bandiere Blu in Italia, Franco Angeli, Milano.

PERRINI F., TENCATI A. (2008), Corporate social responsibility. Un approccio strategico alla gestione d'impresa, Egea, Milano.

POGUTZ S., WINN M. (2013), "Business, Ecosystems, and Biodiversity: New Horizons for Management Research", Organization and Environment, vol. 26, n. 2, pp. 203-229. 
sinergie Vol. 34, N. 99, 2016

POLESE F. (2005), "Risorse e identità locale per la valorizzazione del territorio: un percorso difficile di integrazione sistemica e networking", in Morvillo A., Petrillo C. (a cura di), Sviluppo del territorio: Identità e integrazione - Il caso del Parco Nazionale del Cilento e del Vallo di Diano, Collana IRAT - CNR, pp.103-194.

POLESE F., MELE C., GUMMESSON E. (2014), "Addressing complexity and taking a systemic view in service research", Managing Service Quality: An International Journal, vol. 24, n. 6, pp. 542-544.

PORTER M.E., KRAMER M.R. (2011), “The Big Idea. Creating Shared Value”, Harvard Business Review, vol. 89, n. 1/2, pp. 62-77.

PRAHALAD C.K., RAMASWAMY V. (2004), "Co-creation experiences: The next practice in value creation", Journal of interactive marketing, vol. 18, n. 3, pp. 5-14.

RE P. (2010), "Un modello micro ed uno macro per la valutazione della creazione di valore dei musei e del settore cultura", Sinergie, n. 82, pp. 183-203.

REYNOSO J. (2009), "Values-based Service for Sustainable Business-Lessons from IKEA", Journal of Service Management, vol. 20, n. 4, pp. 473-475.

REYNOSO J., KANDAMPULLY J., FAN X., PAULOSE H. (2015), "Learning from socially driven service innovation in emerging economies", Journal of Service Management, vol. 26, n. 1, pp. 156-176.

RULLANI E. (2005), "Valori d'impresa e conoscenza in un contesto ambientale a complessità crescente", Sinergie, n. 67, pp. 103-122.

RULLANI E. (2010), Modernità sostenibile: idee, filiere e servizi per uscire dalla crisi, Marsilio Editori, Venezia.

RULLANI E. (2012), "Management in transition. Research and action in a world on the move", Sinergie, n. 87, pp. 26-42.

RUSCONI G. (1997), Etica e impresa. Unianalisi economico-aziendale, Clueb, Bologna.

SARRA A., MAZZOCCHITTI M. (2014), "Editoriale: La dematerializzazione dei motori della crescita sostenibile", L'industria, vol. 35, n. 4, pp. 575-588.

SAVIANO M. (2014a), "The role and involvement assigned to businesses in the post 2015 process", in ESDN Conference 2014, A renewed policy framework for sustainable development - The international SD agenda and its impact on Europe, Rome, 6-7 November 2014.

SAVIANO M. (2014b), "Leducation come leva per la transizione verso la sostenibilità", in Settimana del Decennio UNESCO di Educazione allo Sviluppo Sostenibile, 24 novembre 2014, Roma.

SAVIANO M. (2015), "Multi-actor co-creation systems for progressing toward sustainability: criticalities and challenges", in 5th International Conference on Sustainability Science (ICSS), Tokyo, 22-23 January.

SAVIANO M., BASSANO C., CALABRESE M. (2010), "A VSA-SS Approach to Healthcare Service Systems. The Triple Target of Efficiency, Effectiveness and Sustainability", in Service Science, Informs, vol. 2 n. 1/2, pp. 41-61.

SAVIANO M., CAPUTO F. (2012), "Le scelte manageriali tra sistemi, conoscenza e vitalità", in XXXV Convegno annuale AIDEA - Management senza confini. Gli studi di management: tradizione e paradigmi emergenti, Salerno, 4-5 Ottobre.

SAVIANO M., MAGLIOCCA P. (2003), "Programmazione negoziata e governo del territorio. Modelli, tecniche e strumenti”, Esperienze d'Impresa, n. 9, Serie Speciale 2, pp. 163-182. 
SAVIANO M., PARIDA R., CAPUTO F., DATTA S.K (2014), "Health care as a worldwide concern. Insights on the Italian and Indian health care systems and PPPs from a VSA perspective", EuroMed Journal of Business, vol. 9, n. 2, Marialuisa Saviano
Il valore culturale del patrimonio naturale pp. 198-220.

SCIARELLI S. (2003), "Il governo dell'impresa in una società complessa: la ricerca di un equilibrio tra economia ed etica", Sinergie, n. 61-62, pp. 97-115.

SCIARELLI S. (2005), "Lampliamento della responsabilità sociale dell'impresa", Sinergie, n. 67, pp. 35-43.

SEITANIDI M.M, CRANE A. (eds., 2013), Social Partnerships and Responsible Business, Routledge, London.

SEN A. (1986), Scelta, benessere, equità, Il Mulino, Bologna.

SIANO A. (2012), "La comunicazione per la sostenibilità nel management delle imprese", Sinergie, n. 89, pp. 3-23.

SIANO A. (2014), Management della comunicazione per la sostenibilità, Franco Angeli, Milano.

SIANO A., BASILE G., CONFETTO M.G. (2006), "Il ciclo risorse-capacitàcompetenze nell'approccio sistemico vitale: dall'individuo all'organizzazione", in Barile S. (a cura di), L'impresa come sistema. Contributi sullapproccio Sistemico Vitale (ASV), Giappichelli, Torino.

SIMONE C., POLESE F., IANDOLO F., CAPUTO F. (2014), "Alla ricerca di un possibile principio evolutivo della teoria e della pratica d'impresa. Il percorso degli studi delleconomia d'impresa", in XXVI Convegno annuale di Sinergie "Manifattura: quale futuro?", 13-14 Novembre, Cassino.

SPADAFORA S. (2013), "Il rapporto tra uomo e natura: informare, comunicare, formare ed educare", Sinergie Rapporto di ricerca, n. 37, pp. 57-69.

TAMMA M. (2010), "Prodotti culturali e territori: l'immateriale che "vive" nella materialità", Sinergie, n. 82, pp. 27-46

TENCATI A., ZSOLNAI L. (2009), "The collaborative enterprise”, Journal of Business Ethics, vol. 85, n. 3, pp. 367-376.

TENUTA P. (2009), Indici e modelli di sostenibilità, Franco Angeli, Milano.

TEOFILI C., CLARINO R. (2008), Riconquistare il paesaggio. La Convenzione Europea del Paesaggio e la Conservazione della Biodiversità in Italia, WWF Italia ONG ONLUS, Ministero dell'Istruzione.

TOMMASETTI A., SACCOMANNO A. (2012), "Struttura e gestione delle aziende vitivinicole campane legate alla produzione di vini provenienti da vigne storiche, ipotesi di marketing territoriale e piano strategico promozionale". in Autori Vari, Le vigne storiche. Indagine, valorizzazione e protezione delle vigne storiche in Campania, Bari, Imago editrice, pp. 35-66.

UNESCO (1970), What is UNESCO?, UNESCO, Paris.

UNESCO (2001), Universal Declaration on cultural diversity, UNESCO, Paris.

UNESCO (2010), Man \& the Biosphere (MaB). An integrated zonation systems, http:// portal.unesco.org/geography/en/ev.php-URL_ID=8763\&URL_DO=DO_ TOPIC\&URL_SECTION=201.html

UNESCO-DIVISION OF ECOLOGICAL AND EARTH SCIENCE (2014), Future strategy for MaB and the World Network of Biosphere Reserves 2014-2021, UNESCO, Paris.

VAGNANI G. (2005), Ambiente fisico e dinamiche d'impresa: Verso la formulazione di un modello di analisi, Cedam, Padova. 
sinergie italian journal of man Vol. 34, N. 99, 2016

VARGO S., LUSCH R. (2006), "Service-dominant logic: What it is, what it is not, what it might be", in Lusch R., Vargo S. (Ed.), The service-dominant logic of marketing: Dialog, debate, and directions, M.E. Sharpe, Armonk, pp. 43-56.

VARGO S.L., MAGLIO P.P., AKAKA M.A. (2008), "On value and value co-creation: A service systems and service logic perspective", European management journal, vol. 26, n. 3, pp. 145-152.

VARGO S.L., LUSCH R.F. (2010), "From repeat patronage to value co-creation in service ecosystems: A transcending conceptualization of relationship", Journal of Business Market Management, vol. 4, n. 4, pp. 169-179.

VITO G. (1986), "Premesse e condizioni per la formulazione di una strategia aziendale ecologicamente efficace", in Imprese e istituzioni nel governo dell'ambiente: atti del Convegno svoltosi a Lecce, 18-19 settembre 1997, Clueb, Bologna.

WIELAND H., POLESE F., VARGO S., LUSCH R. (2012), “Toward a service (eco) systems perspective on value creation", International Journal of Service Science, Management, Engineering and Technology, vol. 3, n. 3, pp. 12-24.

WORLD COMMISSION ON ENVIRONMENT AND DEVELOPMENT (1987), Our Common Future, Oxford University Press, Oxford.

\section{Riferimenti normativi}

LEGGE 394/91 DEL 6 DICEMBRE 1991, Legge quadro sulle aree protette, pubblicata sulla Gazzetta Ufficiale n. 292 del 13 dicembre 1992.

\section{Siti internet}

www.asvsa.org

www.iucn.it

www.minambiente.it

www.parcosila.it

www.sustainabledevelopment.un.org

www.unesco.it

\section{Academic or professional position and contacts}

\section{Marialuisa Saviano}

Associate Professor of Management

University of Salerno - Italy

e-mail: msaviano@unisa.it

sinergie

ISSN 0393-5108 DOI $10.7433 / \mathrm{s} 99.2016 .10$ pp. $163-190$

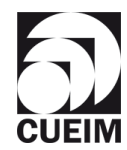

